\title{
«Si una persona tiene más sueños que recuerdos, en su cabeza es joven, independientemente de la edad que tenga»: una conversación con Ernesto Rodríguez
}

\section{Ernesto Rodríguez' entrevistado por Carles Feixa ${ }^{2}$}

Resumen (analítico): Ernesto Rodríguez (Montevideo, 1954) es uno de los referentes latinoamericanos en los estudios y las políticas de juventud. Su interés por la juventud surgió durante su militancia juvenil y maduró durante la dictadura militar uruguaya (1973-1984) en el seno de la oposición democrática. Tras la recuperación de la democracia, Rodríguez fundó el Centro Latinoamericano de la Juventud (Celaju), el cual se convirtió en un think tank sobre estos temas para todo el subcontinente. En los últimos años ha sido el máximo exponente de lo que puede denominarse «juvenología aplicada», asesorando a gobiernos y organismos públicos internacionales en políticas de juventud, sin dejar de investigar y preocuparse por la evolución de las juventudes. La entrevista hace un recorrido por su trayectoria, explica el origen de las instituciones nacionales e internacionales en las que trabajó y contiene un epílogo con una reflexión a distancia sobre los efectos de la pandemia entre las nuevas generaciones.

Palabras clave: juventud, Uruguay, América Latina (Tesauro de Ciencias Sociales de la Unesco).

Palabras clave autores: movimientos juveniles, políticas de juventud, juvenología.

\footnotetext{
${ }^{1}$ Director del Centro Latinoamericano de la Juventud, Montevideo, Uruguay. Correo electrónico: erodrigu1@gmail.com

${ }^{2}$ Catedrático de Antropología Social en la Universitat Pompeu Fabra, Barcelona (Cataluña, España). Correo electrónico: carles.feixa@upf.edu
} 


\title{
«If a person has more dreams than memories in his head, he is young, regardless of how old he is»: A conversation with Ernesto Rodríguez
}

\begin{abstract}
Ernesto Rodríguez (Montevideo, 1954) is one of the Latin American referents in youth studies and policies. His interest in youth arose during his youth militancy and matured during the Uruguayan military dictatorship (1973-1984) within the democratic opposition. After the recovery of democracy, Rodríguez founded the Latin American Youth Center (Celaju), which became a think tank on these issues for the entire subcontinent. In recent years he has been the greatest exponent of what can be called «applied youthology», advising governments and international public organizations on youth policies, while continuing to investigate and worry about the evolution of youth. The interview takes a tour of his career, explains the origin of the national and international institutions in which he worked, and contains an epilogue with a remote reflection on the effects of the pandemic among the new generations..
\end{abstract}

Keywords: Youth, Uruguay, Latin America (Social Sciences Unesco Tesaurus).

Autors keywords: Youth movements, Youth policies, Youthology.

\section{«Se uma pessoa tem mais sonhos do que memórias na cabeça, ela é jovem, não impor- ta a idade que tenha»: Uma conversa com Ernesto Rodríguez}

Resumo (analítico): Ernesto Rodríguez (Montevidéu, 1954) é um dos referentes latino-americanos em estudos e políticas juvenis. Seu interesse pela juventude surgiu durante sua militância juvenil e amadureceu durante a ditadura militar uruguaia (1973-1984) dentro da oposição democrática. Após a recuperação da democracia, Rodríguez fundou o Centro da Juventude Latino-Americana (Celaju), que se tornou um think tank sobre essas questões para todo o subcontinente. Nos últimos anos, ele tem sido o maior expoente do que se pode chamar de «juventude aplicada», assessorando governos e organismos públicos internacionais sobre políticas de juventude, ao mesmo tempo em que continua investigando e se preocupando com a evolução da juventude. A entrevista faz um tour pela sua carreira, explica a origem das instituições nacionais e internacionais em que atuou e contém um epílogo com uma reflexão remota sobre os efeitos da pandemia entre as novas gerações.

Palavras-chave: Juventude, Uruguay, América Latina (Tesaurus de Ciencias Sociais da Unesco).

Palavras-chave autores: Movimentos juvenis, Politicas da juventude, Juvenologia. 


\section{Introducción}

Ernesto Rodríguez (Montevideo, 1954) es uno de los referentes latinoamericanos en los estudios y las políticas de juventud. Nos conocimos en México en 1998, cuando participamos en el encuentro preparatorio de la Encuesta Nacional a la Juventud de México, invitados por José Antonio Pérez Islas. Antes habíamos coincidido en Barcelona en 1985, durante el congreso que tuvo lugar con motivo del Año Internacional de la Juventud, aunque en ese momento no nos conocimos, puesto que yo estaba acabando mis estudios y participé en el evento como delegado del Consejo Nacional de la Juventud de Cataluña (seguramente ese evento fue la causa de que luego me dedicara a la investigación sobre juventud). Desde entonces hemos coincidido varias veces en congresos y encuentros por toda América Latina y también en España (Ernesto ha sido siempre un viajero impenitente). Su interés por la juventud surgió a los 17 años, en el seno de las organizaciones cristianas progresistas (como otros juvenólogos), y maduró durante la dictadura

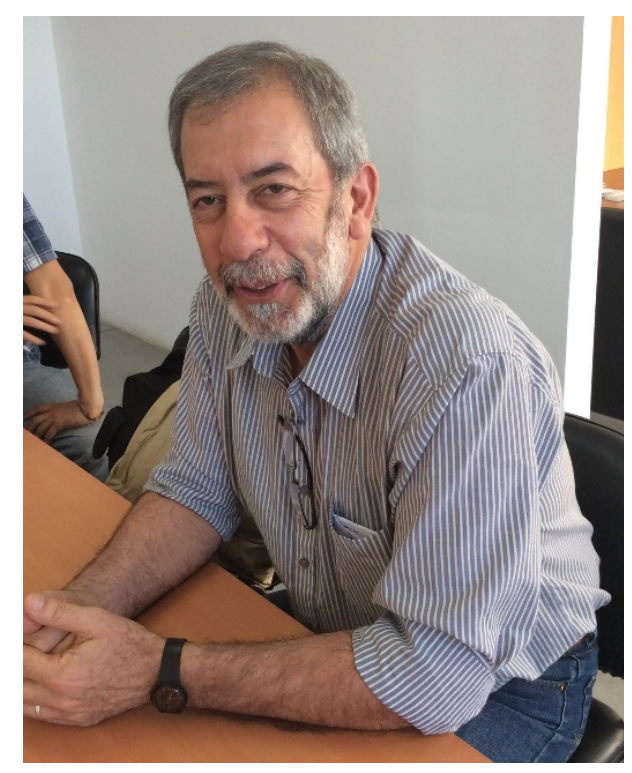

Ernesto Rodríguez durante la entrevista en La Plata, Argentina (22-10-2014). Foto (c) Carles Feixa militar uruguaya (1973-1984) en el seno de la oposición democrática (en concreto del Frente Amplio). Tras la recuperación de la democracia, Rodríguez fundó el Centro Latinoamericano de la Juventud (Celaju), el cual se convirtió en un think tank sobre estos temas en todo el subcontinente.

En los últimos años Ernesto ha sido el máximo exponente de lo que puede denominarse «juvenología aplicada», asesorando a gobiernos y organismos públicos internacionales en políticas de juventud, sin dejar de investigar y preocuparse por la evolución de las juventudes. En sus publicaciones ha abordado temas como los movimientos sociales, la violencia, la educación, los índices de desarrollo, las políticas públicas y las tribus urbanas (ver su amplia bibliografía al final). La entrevista se lleva a cabo a fines de 2014 en el hotel donde nos alojamos, al final del congreso Jumic sobre Jóvenes, medios de información y comunicación, organizado por 
Florencia Saintout en la Universidad de La Plata, Argentina. Contiene un epílogo con una pregunta sobre la situación actual, con respuesta a distancia en plena pandemia.

\section{Cosecha del 71}

Carles Feixa (C. F.): De entrada, si puedes presentarte...

Ernesto Rodríguez (E. R.): Soy Ernesto Rodríguez, uruguayo, sociólogo. Actualmente trabajo en consultorías internacionales para América Latina, siempre en el campo de las políticas públicas de juventudes. Llevo en esto como 35 años; un pequeño recorrido de todo esto pasa por la suma de un montón de casualidades. Nací en Montevideo en el año 1954, tengo 6o años; en este momento y para mí una de las fechas importantes fue el año de 1971, año de elecciones nacionales en Uruguay; las últimas, antes del golpe de estado de 1973. En el año 71 yo tenía 17 años y estuve entre los fundadores del Frente Amplio, que se creó en ese año para participar en esas elecciones y a mí me pareció realmente muy interesante la propuesta que se hacía y me incorporé a trabajar en este nuevo (entonces) espacio político, con 17 años de edad. El ${ }_{71}$ fue un año muy complicado porque venía precedido de una etapa muy particular, unos años sesenta muy convulsionados: una guerrilla muy activa en Uruguay, un movimiento sindical muy activo, un régimen autoritario desde el 68 y una crisis económica muy grande.

El Frente Amplio se constituye para tratar de dar alternativas a todas estas cosas. Entonces las elecciones no resuelven nada a los conflictos planteados eventualmente, y en el año 73 se da el golpe de Estado por los militares; un golpe muy particular porque es el propio Presidente de la República quien clausura el Parlamento junto con los militares y continúa en su cargo. Las elecciones en ese año las gana el Partido Colorado, pero había un proyecto de reforma constitucional en paralelo a la elección; si lograba la mayoría de votos ganaba quien iba como candidato presidencial, pero si no se lograba esa mayoría, ganaba el que estaba como candidato a vicepresidente, que fue lo que eventualmente ocurrió. Quien gana es Juan María Bordaberry, que da el golpe de Estado con los militares. Un año en el cual la guerrilla urbana estaba ya prácticamente derrotada; en el año 72 hubo una guerra interna muy grande, y había ya una gran movilización social, sindical, política, etc.; así que se decide que finalmente hay que eliminar el Parlamento y empezar un marco dictatorial. Esa dictadura duró doce años, que coincidieron con mi juventud y fue un periodo bastante complejo.

Nosotros nos pusimos a trabajar en el campo de la oposición a la dictadura, en el caso mío organizando movimientos juveniles en la resistencia en un marco de partidos políticos y movimientos sindicales y estudiantiles ilegalizados, y donde apenas podía haber un marco de actividad 
de la Iglesia Católica que en esa época tenía ideas muy progresistas. Varios de los curas y obispos se adherían directa o indirectamente al marco de la teología de la liberación, lo que era muy interesante, porque también se ubicaron en el campo de la oposición no de manera ostentosa, pero sí colaborando con los grupos opositores, brindando sus espacios, sus iglesias, para que se pudieran hacer actividades de todo tipo en esta materia. En el caso nuestro nos tocó organizar muchos grupos juveniles a lo largo y ancho del país en un contexto donde todo estaba prohibido... desde la clandestinidad.

El Frente Amplio era una coalición de partidos; básicamente, el Partido Comunista (el más relevante de América Latina en esa etapa), el Partido Socialista y el partido del que yo formaba parte, el Partido Demócrata Cristiano, que a diferencia de muchos otros tiene ideas progresistas muy avanzadas. Nuestro modelo de sociedad era el socialismo comunitario (algo de lo que habla ahora Evo Morales) y nuestro rol fue clave en la convocatoria a la conformación del Frente Amplio, a donde llegaron también algunos grupos de los partidos tradicionales: el Partido Nacional y el Parido Colorado. El salto fue muy importante, porque la izquierda nunca había pasado del 5\% de los votos y con la construcción del Frente Amplio llegó al 18\%; así se rompió el bipartidismo tradicional que venía con una hegemonía de más de cien años.

La dictadura fijó reglas de juego diferentes para todos, para cada uno de los sectores de oposición. Clasificó a toda la ciudadanía en tres categorías: A, B y C. Los de la categoría A eran los que no tenían ningún problema con el régimen, a los de la categoría $\mathrm{B}$ había que vigilarlos, eran muy sospechosos, y los de categoría C eran los enemigos. Por supuesto, en su momento nadie se enteró en qué categoría estaba. Nos enteramos después que terminó la dictadura porque a uno le negaban o no un pasaporte aludiendo que uno pertenecía o no a alguna de estas listas. Y en ese contexto, con el paso del tiempo nos fuimos enterando que nuestro partido, el PDC, estaba en la categoría intermedia y en mi caso, por algo de lo que me enteré tarde, porque yo era un líder juvenil importante en la época y un opositor sensato, según decía un informe.

C. F.: ¿Pudiste ver los informes?

E. R.: Sí, después que se recuperó la democracia. La recomendación que hacía era que había que vigilarme muy estrictamente y controlar mis movimientos; que no convenía meterme preso o desaparecerme..., porque tenía muchos amigos en el exterior, algo que estaba un poco exagerado. Es cierto que en esa época yo trabajaba en el área de relaciones internacionales del partido. 
Yo hice mi licenciatura en Sociología. Mi primer libro fue en temas de juventud en el año 78 , luego siguió otro en ciencias políticas en temas internacionales y me dediqué a trabajar en eso; algo que luego transformé en uso periodístico. En esa etapa yo trabajaba mucho en contactos con el exterior, viajaba muchísimo a Europa donde teníamos varios aliados en aquella época, en Italia, Bélgica, Holanda y Alemania; por eso tenía apoyo relevante internacional, y cada vez que volvía de esos viajes me detenían, me preguntaban toda clase de cosas, me averiguaban, llegaba desde las embajadas uruguayas toda clase de entrevistas que yo hacía a la prensa de esos países y con eso se fue acumulando un montón de papeles que yo pude conocer recién cuando se restableció la democracia.

En los primeros años la dictadura fue algo muy feroz, dedicada a destruir al enemigo; de alguna manera salieron muchos uruguayos al exilio, otros tantos terminaron presos, otros tantos desaparecidos; aunque no ha tenido la dimensión de otros países (en Argentina, sin ir más lejos). Algunos pocos pudimos seguir haciendo actividad política en las márgenes; era cuanto se podía aspirar en aquellos años: 73, 74, 75, 76, que fueron años muy complejos. Luego la dictadura evaluó que la limpieza ya se había logrado y comenzó una etapa de estabilización del régimen. En la economía empezaron a ponerse en práctica medidas que hoy conocemos como neoliberales; la economía se abrió al mundo, empezaron a verse ciertas privatizaciones y, en dicho marco, se comenzaron a flexibilizar algunos espacios de participación vigiladas y algunos sectores empezamos a lidiar con los temas más espinosos, tratando de hacerlo de manera más abierta.

\section{Situación y perspectivas de la juventud uruguaya}

Y la primera decisión que se tomó es empezar a escribir cosas, con todas las censuras correspondientes, y mi primer libro fue una mezcla de lo que trasmitía en orales, talleres, cursos y encuentros que hacíamos con todos los jóvenes de todo el país: Conjunciones y perspectivas de la juventud uruguaya, que se publicó en el Centro de Investigación de Economía Humana (CLAEH), fundado bajo la inspiración del padre Lebret de Francia, de amplia influencia en las corrientes progresistas de la Iglesia Católica a nivel mundial, por lo que se fundaron varios centros similares en varias partes del mundo. En América Latina se trabajó en Montevideo todo ese conjunto de cosas y ese Centro tenía la opción de publicar informes muy generales y empezamos a dar informes de la situación económica, algunos más concentrados en sectores sociales y otros temas afines.

C. F.: ¿Cómo surgió ese estudio? Dijiste que había una serie de casualidades. ¿Fue una idea tuya, un encargo? 
E. R.: Fue una iniciativa colectiva, diría yo. Hasta ese momento se trasmitían mensajes oralmente, en pequeños grupos, pero llegó un momento que la demanda era tal que hubo una necesidad de comenzar a transmitirlos de otra manera (grabaciones, textos escritos, etc.). Esa fue una de las razones fundamentales. En paralelo yo había comenzado a estudiar economía en la universidad. Cuando comencé con estos temas varios amigos me convencieron que era mejor que estudiara sociología y me dedicara más de lleno a este tipo de cosas, más académicamente. Yo entré a la universidad en el año 72; en el año 73 fue el golpe de Estado y se intervino la universidad. Yo estaba estudiando economía y el decano de la facultad en ese momento era el actual vicepresidente de la república; el rector era otro economista muy importante con el que tenía un vínculo muy fuerte y que en los años noventa asumió la titularidad del Ministerio de Educación.

Yo militaba en esa época como dirigente estudiantil y terminamos presos todos: rectorado, decanatos, dirigentes estudiantiles, todos juntos; entonces tenía un vínculo muy fuerte con quienes trabajábamos (muchos de ellos pasaron a dar clase en el CLAEH) y se comenzó con una serie de publicaciones muy elementales, evidentemente estenografiadas: Las primeras eran reseñas y análisis históricos, ubicados muy lejos en el tiempo, cosas que no generaran problemas o reuníamos evidencia de algún tema en particular. Ese fue mi primer informe que quise hacer, algo más terrenal y, bueno, se publicó. Tuvo bastante buen eco y nos ayudó mucho en el trabajo de promoción juvenil que estábamos realizando, porque permitió ampliarlo mucho y comenzó a permitir generar ciertos grupos con este tipo de reflexiones, con la gente que se dedicaba a este tipo de cosas en muy diversos puntos del territorio nacional.

C. F.: ¿En qué te basaste para hacerlo?

E. R.: Por un lado, en el trabajo de conocer la realidad del país con los jóvenes, por aquí y por allá; y, por otro lado, revisar antecedentes de literatura disponible, realizar encuestas donde uno podía recuperar datos relevantes en algún sentido, etc. Fue un primer informe muy general, con datos sobre las matrículas educativas, sobre las tasas de empleo, sobre algunas cuestiones en salud. En ese momento teníamos un problema muy grande de migración y yo me encontré con un texto que me ayudó mucho, bastante original para la época, hoy ya un clásico: Introducción a la sociología de la juventud (Allerbeck \& Rosenmayr, 1979). Y en América Latina había un texto muy interesante de Aldo Solari (Algunas reflexiones sobre la juventud latinoamericana), que había publicado el Ilpes en 1971. Estos dos textos eran referentes en ese momento, pero no había mucha otra cosa por el estilo en América Latina; lo máximo que teníamos eran estudios sobre movimientos estudiantiles del año 68, pero no mucho más. Implicó que ese primer informe de unas cien páginas terminara llenando un vacío muy importante en el momento y si- 
gue siendo un referente (ahora clásico) para todo lo que se ha hecho en estos temas desde entonces.

El otro año clave fue 1980 , por muchas cosas: en lo político porque la dictadura pretendió aprobar una reforma constitucional para legitimar o legalizar la dictadura, en un modelo muy corporativo en el que los partidos políticos desaparecían como tal y pasaban a cumplir otras funciones muy genéricas. Era un planteamiento corporativo de corte fascista muy claro. En la oposición trabajamos por el No muy intensamente y logramos participar en la consulta con muchos partidos inscritos sin usar los nombres tradicionales (se le daba a cada grupo letras, A, B, C, etc., porque la idea era radicar de la memoria de la gente los partidos políticos que había previamente) y, aún en un contexto donde la dictadura controlaba los medios de comunicación, se logró revertir esa propuesta de la dictadura y triunfó el No con el $57 \%$ de los votos, con lo que se abrió la etapa de transición a la democracia. El principal error del grupo que estaba en el gobierno fue el no pactar con ningún partido, la propuesta era antitodos.

Pero 1980 también fue clave porque es el año en que yo me casé y para mí eso en mi vida es un corte muy grande; el cura que nos casó dirigía el Servicio de Paz y Justicia, estábamos en plena dictadura; nos casamos en marzo y después tuvimos el plebiscito en noviembre. La iglesia donde nos casamos se llenó de dirigentes políticos y opositores grabando toda la ceremonia. En un momento pensamos que la luna de miel la pasaríamos en prisión, pero finalmente no ocurrió nada y mi vida comenzó desde allí otra etapa totalmente diferente. Llevo 35 años viviendo con la persona que me casé y no he tenido cambios en esa materia, por lo que, desde siempre, muchos amigos me ven como un marciano.

\section{El Foro Juvenil}

A raíz del plebiscito tomamos otra decisión importante en materia de levantar limitaciones y empezamos a trabajar dando la cara y nos decidimos a crear una ONG; creo que vale la pena decir cómo se produjo todo esto, porque es muy interesante. El Foro Juvenil lo fundamos en marzo del 81, mientras que el Celaju lo creamos en el 86. Como ya comenté, me caso en marzo de 1980 y en julio me invitan a un encuentro en Estrasburgo y me encuentro con un amigo que estaba estudiando en Bélgica y nos ponemos a conversar. Era compañero mío de militancia política, además de ser un gran amigo; nos ponemos al día de cómo estaban las cosas en Uruguay y él me dice que le parece increíble que yo esté haciendo las cosas que estoy haciendo, que esté movilizando tantos jóvenes en el país, en medio de la situación que estábamos viviendo en ese momento. Acto seguido, me hace una pregunta que en su momento me sorprendió mucho (hoy entiendo que fue una pregunta totalmente pertinente): «¿Quién te financia todo 
este trabajo?», y le respondo que es un trabajo estrictamente militante y que no recibo ningún tipo de apoyo económico, que sacábamos recursos de nuestro propio bolsillo para imprimir algún volante o alguna otra cosa; y él me dice: «Qué bueno», pero le costó creerlo hasta que se convence y luego dice que podíamos conseguir algunos recursos para trabajar con un poco más de holgura. En ese momento no teníamos todavía la idea de crear la ONG; teníamos la idea de trabajar más porque teníamos que impulsar el plebiscito. Me dijo que si me parecía bien me podía quedar otra semana más en Europa y él mientras tanto hace unas llamadas telefónicas, para armar una agenda de visitas con ese fin. Me comentó que podía conseguir recursos para trabajar con un poco más de margen; le dije que sí y nos fuimos para su casa y una de las visitas claves que hicimos fue al presidente del Consejo Comunal de Iglesias, en Ginebra, Suiza, que era otro uruguayo, un pastor protestante, otro personaje muy importante en mi vida y en mi carrera profesional, sin duda.

Cuando le contamos lo que estaba haciendo, él me hace la misma pregunta que mi amigo, pero él tenía una gran ventaja respecto a mi amigo y es que tenía el poder de solucionarlo. Y entonces me dice: «pero es muy importante lo que usted hace». En ese momento, este Consejo de Iglesias (que agrupaba a todas las iglesias de Europa y del mundo) era muy importante, y en su oficina, el Pastor Castro hace unas llamadas y nos dice: «iSe animan a ir a Zeist, en Holanda, para que hablen con fulanito de esta organización?», una fundación de iglesias evangélicas, y dijo: «Todos aquí sabemos que vamos apoyar una iniciativa político partidaria y dadas las circunstancias tenemos que hacer eso, pero no podemos hacerlo formalmente». Ahí surge la propuesta que formemos una ONG; mi amigo dice que sí, que no hay ningún problema. Pero yo no tenía ni la menor idea de lo que era una ONG. Nos despedimos, tomamos un tren; en Europa los trenes eran increíblemente puntuales, pero como la cita con el señor era 15 minutos después que llegaba el tren a la ciudad, yo pensaba que, si se demoraba..; pero mi amigo me decía que no me preocupara, que «aquí los trenes no se demoran», algo que pude comprobar regularmente desde entonces y hasta ahora, por cierto.

C. F.: ¿Era tu primer viaje a Europa?

E. R.: No, el primero fue en 1978, invitado por la Fundación Konrad Adenauer de Alemania Federal (en ese entonces). Nos tomamos un tren y en el camino mi amigo me explica qué es una ONG y entonces empezamos a buscarle un nombre, hasta que llegamos a Foro Juvenil, que reflejaba muy bien lo que estábamos haciendo. Esta ONG necesitaba un director, que obviamente era yo; necesitaba una dirección, un teléfono y pusimos la de una academia donde nosotros preparábamos a estudiantes de secundaria para sus exámenes, que era una forma de ganarnos la vida en ese momento. Mi amigo me dice: «Vení», y me sienta frente a una máquina y 
me dice: «Necesitamos que el director de Foro Juvenil tenga una tarjeta de presentación»; fue la primera tarjeta de presentación que tuve en mi vida (impresa allí, en la estación de trenes). Cuando llegamos nos presentamos, entregué mi tarjeta de presentación y echamos todo el cuento. Viene entonces la pregunta clave: «¿Cuánto precisa?». Yo no tenía la menor idea de qué responder, por lo que mi amigo dice: «A corto plazo cuarenta mil o cincuenta mil dólares», y de allí entramos al plebiscito de la época. Yo me limité a asentir con la cabeza y ahí surgió el apoyo a nuestra iniciativa, sin que yo pudiera salir de mi asombro, por cierto.

C. F.: ¿Cuál era el objetivo?, ¿movilizar a los jóvenes?

E. R.: Allí surgieron las primeras orientaciones: «Necesito que ustedes aparezcan en un proyecto, en este formato, que hagan una demanda para este año y el siguiente, por cien mil dólares, y yo me encargo de movilizarlo; ustedes me lo envían y yo me encargo de responder rápidamente; pero cuenten con esto, incluso si tienen deudas mientras llegan los recursos para comenzar todo esto». Hubo una gran apertura a colaborar sin duda, el mismo 1980; hago todas estas gestiones y se concretaron los pasos anunciados, al pie de la letra. En la misma época, se creó en Brasil el Partido de los Trabajadores, el partido de Lula da Silva, y coincidió con que desde allí también llegaron a las mismas puertas a pedir también recursos para cosas muy parecidas. Por ello, también se creó una ONG en Brasil y también le dieron el apoyo correspondiente y entonces comenzamos a tener un intercambio muy fuerte desde el origen mismo con el Partido de los Trabajadores de Brasil (visitas recíprocas, etc.)

Cuando recibo la notificación de que el proyecto estaba aprobado, me dijeron que debía tener una cuenta bancaria, y en mi vida nunca había tenido una cuenta bancaria, ni nunca había visto más de veinte o treinta dólares juntos. Tuve que hacer mi cuenta bancaria y de la noche a la mañana tenía una cuenta con cien mil dólares, con lo cual dijimos, ahora vamos a ponernos a trabajar y finalmente fundamos e instalamos el Foro en marzo, después del plebiscito que había ganado la oposición y tocaba empezar a dar la cara para forzar al máximo la apertura. En marzo del 81 nos proponemos formar un consejo directivo; le entramos de lleno al armado de los programas, empezamos a hacer más boletines informativos, cursos, talleres, encuentros, y se trasformó en la instancia o el espacio en que se recreaban o generaban movimientos juveniles en Uruguay; desde allí recreamos el movimiento estudiantil y la rama juvenil de los sindicatos. Uno de los protagonistas de esa época estudiaba medicina con mi esposa; mientras yo trabajaba con los movimientos juveniles; él trabajaba con los grupos juveniles de los sindicatos, $\mathrm{y}$ juntos fuimos a un Encuentro Internacional, Incontro per il diritto dei giovani uruguaiani a vivere nella loro patria, en el que se reunieron jóvenes uruguayos que estaban en el exilio, junto a 
otros jóvenes europeos que estaban respaldando los procesos de restauración democrática en nuestro país. Esta era otra dimensión de gran relevancia para nosotros, sin duda.

C. F.: ¿La investigación sobre juventud qué papel tuvo en esa fundación?

E. R.: Fue bastante azaroso. Yo después que hice mi licenciatura publiqué mi primer libro, me contrataron para hacer otro y seguí investigando del tema ya más profesionalmente. Hice después un texto sobre jóvenes rurales y después hicimos algo muy importante que fue empezar a difundir información sobre diversas áreas específicas, porque toda la información disponible estaba muy dispersa. El mejor ejemplo que tuvimos — creo- fue un texto que publicamos en el 82 a propósito de la matrícula en secundaria. Hasta ese momento, teníamos solo lo que había sobre el tema, que eran los resultados de los dos censos que habíamos tenido en 1963 y 1975 y en la comparación de esos censos, la matrícula crecía; y cuando logramos que un amigo que trabajaba en estadística en el área de secundaria del Ministerio de Educación de la dictadura nos diera los datos de la matrícula del Ministerio (que eran reservados) resultó que la matrícula crecía hasta el golpe de Estado (año 73) y, a partir de allí, comenzaba a bajar drásticamente. Nosotros publicamos esa información y eso generó tres impactos muy grandes: uno en la opinión pública, que comenzó a generar conciencia de un problema del que no se conocía nada; dos, en el campo académico, ya que los que estudiaban educación no habían tenido acceso a esa información, por lo que todo lo que se decía de la educación no tenía nada que ver con la realidad; y, tres, tuve problemas con los servicios de seguridad: «iDe dónde sacaron esos datos?», «¿quién se los dio?»...; seguíamos todavía en dictadura.

\section{La cosa es que empiezan a hacer preguntas}

Creo que fue allá por 1980. Yo vivía en una cooperativa de viviendas de ayuda mutua y hasta allí llegó el ejército, parando frente a las puertas de mi casa. Lo que calculamos era que venían para llevarme preso otra vez (era muy reiterado, eso ocurría cuatro, cinco y seis veces al año por periodos de una semana, a veces tres); pero llegaron a casa muy cordialmente, preguntando por el señor Ernesto Rodríguez y me entregan una invitación, creo que del Jefe de la Dirección Nacional de Información e Inteligencia, invitándome a que presentara el libro ante la oficialidad; y en paralelo van al instituto donde había publicado el libro (el CLAEH) y compran 300 ejemplares. La secretaria del instituto era mi hermana y enseguida me llama y me dice que no lograban salir del asombro y que nadie entendía nada. La cosa es que se concreta esa reunión, me presentan como un opositor al régimen; habían estudiado el tema de los jóvenes y piden que me pongan mucha atención. Yo doy mi exposición muy cargada de datos estadísticos para tratar de atemperar los posibles impactos; trato de evitar valoraciones y juicios, pero en la 
ronda de preguntas, comenzaron a acumularse las preguntas más ideológicas y políticas, lógicamente.

C. F.: ¿La reunión fue de jefes militares o soldados?

E. R.: De la oficialidad media y superior. En esa época para mí todo era muy nuevo, hasta las preguntas que me hicieron: «iA los jóvenes que están vinculados al movimiento subversivo, a la guerrilla Tupamara, los denunciaría o no los denunciaría?», yo respondía haciendo un análisis de los jóvenes como en conjunto. «Usted apoya a jóvenes que no tienen empleo, que tienen dificultades en educación, en concreto, ¿qué haría?», y cosas de ese tipo. Fue bastante compleja la situación, pero bueno, logré salir por la puerta por donde había entrado y eso ya era un logro importante. Comento esto para ilustrar que la investigación que hacíamos se fue cargando de ese tipo de estudios, muy en función de cosas muy concretas en términos del proceso político. No fue una investigación académica clásica, pura, y esta fue la tónica en el 83, el 84, el 85 . Clacso estaba por esa época estudiando los movimientos sociales, haciendo seminarios y libros por país sobre estos temas, y un sociólogo muy importante, Carlos Filgueira, coordinó el libro que se hacía sobre el caso uruguayo y me pidió que hiciera el capítulo sobre movimientos juveniles; entonces fue otra oportunidad para poder seguir con el tema desde otro tipo de ángulo.

Yo había estado continuamente con los movimientos juveniles, pero creo que lo más relevante fue comenzar a trabajar en la propuesta para restablecer la democracia. Entonces, en los años 83 y 84 -si no me equivoco- se instala en Uruguay algo muy importante que se llamó la Concertación Nacional Programática, con el objetivo de que todos los grupos sociales y políticos trataran de ponerse de acuerdo en todo lo que iba a ser la gestión del primer gobierno democrático, independientemente de quien ganara las elecciones. Todavía no se sabía bien cuándo iban a ser las elecciones, por lo que los diálogos eran tan complejos como inciertos, y en ese contexto se armaron grupos de salud, empleo, mujeres, desarrollo rural; en fin, lo clásico en ese tipo de procesos, y nosotros propusimos que hubiera un grupo sobre juventud que me tocó dirigir y entonces armamos las propuesta para el gobierno democrático, para que una vez que hubiera elecciones y se instalara el elenco ganador, se pudiera empezar a trabajar conjuntamente.

\section{5: Año Internacional de la Juventud}

Por muchas razones otro año clave fue 1985 , declarado Año Internacional de la Juventud por las Naciones Unidas. Desde el 1983 se formularon propuestas para que los países generaran comités nacionales de coordinación para celebrar el Año Internacional de la Juventud. Nosotros armamos un Comité en Uruguay; me tocó ser el presidente y a partir de entonces trabajamos mucho en ese sentido. Las elecciones finalmente fueron en el 84 y las gana el Partido 
Colorado y comienza una etapa de restauración conservadora en 1985 . Fue entonces que nosotros nos dedicamos a generar foros con las autoridades electas a nivel nacional y municipal para discutir sobre las propuestas que habíamos elaborado. Se trabajó mucho en esa lógica y se lograron, digamos, algunos avances importantes, pero nos costó mucho. Ese primer gobierno $(85-89)$ fue un gobierno conservador y no se hicieron grandes cosas; hubo una Comisión Coordinadora de la Juventud en el ámbito del Ministerio de Educación (que también impulsamos nosotros), pero allí quedó todo, sin grandes avances sustantivos.

Hasta allí, yo tenía una militancia política muy intensa, hasta los años previos a las elecciones. Pero importa consignar que otro año clave para mí fue el 83, porque se empieza el proceso de construcción de la alternativa democrática, nace mi primer hijo y con mi esposa valoramos que si seguíamos con el ritmo que teníamos, el niño iba a ver a sus padres solo en fotos (mi esposa viajaba mucho igual que yo). Entonces tomamos dos decisiones muy trascendentes: ella decide dejar de viajar, lo que implica dejar un cargo internacional que tenía (trabajaba con niños y adolescentes en el ámbito de la Iglesia Católica) y yo decido dejar la militancia política, en un contexto donde varios partidos me habían propuesto que yo formara parte de la lista de diputados para acceder al Parlamento. Mis amigos no entendían nada: cómo me había bancado los doce años de dictadura y ahora, que podíamos empezar a cosechar todos aquellos esfuerzos, dejaba todo a un lado. Lo último que hice en términos políticos fue estar en el estrado principal del primer gran opositor (que fue también en el año 83), donde se reunió a toda la dirigencia política y social opositora y que para mí fue un motivo de mucho orgullo que me llamaran a formar parte de las 150 personas que había en el estrado, que eran los máximos dirigentes políticos del país.

C. F.: ¿Como representante del foro juvenil?

E. R.: No, como parte de mi militancia política, pero en reconocimiento de mi trabajo con jóvenes. Fue lo último que hice, un acto muy grande, muy publicitado, y los militares después sacaron una lista con los que estábamos, diciendo que éramos todos subversivos, comunistas, opositores al régimen, a la democracia, todos enemigos de la patria, en fin. Pero llegaron los diálogos, llegaron las elecciones, la instalación del gobierno democrático en el año 85, y ahí comenzó otra etapa. Evidentemente yo creo que otra cuestión clave es que el 85 es el año en que se restablece la democracia en Uruguay, el Año Internacional de la Juventud y la Reunión Latinoamericana y del Caribe se hace en Montevideo; lo hicimos con la Cepal que era el organismo de las Naciones Unidas que organizaba formalmente la actividad.

Luego viene el Congreso Internacional en Barcelona, donde me pasaron dos cosas muy importantes. Para ubicarnos en los parámetros políticos de la época, el presidente del Comité 
Internacional de Juventud de Naciones Unidas era el Ministro de las Juventudes de Rumanía, el hijo de Ceaușescu (que cuatro años después terminó como terminó en la plaza con toda su familia); pero el funcionario de Naciones Unidas que trabajaba a cargo de ese Comité Mundial de Juventud era un chileno, Ernesto Ottone, quien se acercó y se presentó (el ministro de Rumanía y él eran los personajes de la reunión), me hizo varios reconocimientos por el trabajo que estábamos haciendo (mostró bastante conocimiento de lo que yo estaba haciendo) y a renglón seguido se presenta, diciendo que había sido presidente de las Juventudes Comunistas en Chile; había sido presidente de la Federación Mundial de Juventudes Democráticas en su momento;

luego, cuando el Partido Comunista decide integrarse a la lucha armada en Chile para combatir a Pinochet, él se va del Partido Comunista y comienza a acercarse a líderes políticos como Ricardo Lagos, y me explica que está exiliado, que no puede volver a Chile, pero quería estar más cerca de Chile y me termina proponiendo: «¿Por qué no hacemos algo desde Montevideo?», del tipo de cosas que estaba haciendo en Uruguay pero para América Latina. Inmediatamente me presenta otro buen amigo, Federico Mañero, español que en esa época trabajaba en relaciones internacionales en el Partido Socialista. Quico Mañero me dice que está organizando la cooperación española para América Latina, que él está organizando la Cooperación Española en Juventud y que le encantaría poder montar un Centro en América Latina, en sintonía con los planteos de Ottone.

\section{La creación del Celaju}

Fue así que el Centro nace con el apoyo de algunos organismos de Naciones Unidas y de la cooperación española. Ellos van a Montevideo (Quico y su esposa, Elena Valenciano), en ese momento trabajaban mucho con movimientos de mujeres, por lo que también nos fuimos metiendo en estos temas de género. Hicimos algunas actividades en torno a estos temas y, en 1986, organizamos un seminario en Montevideo para constituir formalmente el Celaju; ahí invitamos a muchos de los amigos, investigadores que estaban vinculados con estos temas de juventudes, muchos eran discípulos o alumnos de los cursos que daba en Francia Alain Touraine, y ahí aparecieron varios chilenos (Javier Martínez, Ricardo Solari); llegó Sergio Zermeño de México, que conocí antes de conocer a José Antonio y algunos otros, argentinos, brasileños, peruanos...; aparecieron con Germán Rama: Cecilia Braslavsky de Argentina; Felicia Madeira, de Brasil; Rodrigo Parra, de Colombia; Julio Cotler de Perú y varios más. Así fue que el primer equipo que empezó a trabajar en Cepal luego colaboró con la fundación del Celaju, al igual que el director nacional de la juventud de España que venía del Partido Socialista de Cataluña, quien nos plantea que quiere comenzar a trabajar sobre políticas públicas de juventud en América Latina. 
La primera cosa que hicimos con el Celaju fue organizar en el 87 una reunión de investigadores que hicimos en Buenos Aires (fue previa a la que hicimos en México en el 88) y, en paralelo, convocamos a una consulta que hicimos con movimientos juveniles, con apoyo de la Unesco, la que más nos apoyó en esa época. Entonces instalamos la Red de Investigadores de América Latina, así como la Consulta Colectiva con movimientos juveniles, en ambos casos con apoyo de la Unesco y con el apoyo del Instituto de la Juventud de España. Empezamos a hacer reuniones de estas dos redes y convocamos también a una primera conferencia de gobiernos que trabajaran la juventud. La primera reunión fue de gente que venía de Ministerios de Educación, Trabajo, Deporte... Por entonces había solo tres países que tenían Ministerio de la Juventud (México, Costa Rica y Venezuela). La primera la hicimos también en Buenos Aires, la siguiente en Madrid en el 88, la siguiente en Costa Rica en el 89, hasta que finalmente en el 92 se fundó la OIJ en España, sobre la base de todos estos antecedentes. El Celaju fue vital para crear estas tres redes que comenzaron a darle vida en América Latina. En términos sustantivos, nos pusimos a hacer balances de políticas de juventudes en cada país; ya habíamos empezado antes a hacerlo con la Unesco, donde hice mis primeros balances de políticas de juventud en Venezuela, Ecuador, Argentina, por esa época $(87,88)$, y nos dedicamos a sacar algunos boletines informativos latinoamericanos; trabajaba mucho con Héctor Castillo en esa época, y ahí comienza la instalación de las redes y luego termina siendo lo que son hoy en día, más conocidas. Yo creo que otro momento importante es cuando desde el Instituto de Juventud, ya con Magdy Martínez, director del Injuve, se decide hacer el primer informe de juventudes en América Latina, que lo hicimos en el año go y lo publicamos en el 91; fue el primer informe grande que se publicó en América Latina. Uno lo mira en perspectiva y creo que fue uno de los mejores, basado en la literatura disponible y en estadísticas que sobre todo había en Cepal (datos demográficos, encuestas de hogares, etc.).

\section{De los Foros Latinoamericanos de la Juventud a la Cepal}

C. F.: ¿Cómo pasaste de lo nacional a lo internacional, tanto en lo teórico como en lo metodológico?

E. R.: Fue una combinación: por un lado, la invitación de Ernesto Ottone para armar un espacio de trabajo latinoamericano y, por otro lado, yo empecé a tener dificultades con el Foro Juvenil. Yo había dejado la militancia política y había empezado a sostener que —como estábamos, ya en democracia- el Foro tenía qué abrirse más ampliamente, integrando a jóvenes de otros partidos políticos, mientras que varios de mis compañeros entendían que había que seguir como siempre y que yo tenía que dar un paso al costado. Acordamos una ruptura civili- 
zada y ahí comenzó otra etapa. La oficina nuestra en Montevideo pasó a ser la oficina de los chilenos en el exilio y teníamos de canciller a Enrique Iglesias a dos cuadras de la oficina nuestra, que era un gran amigo y nos apoyaba en todo; él fue quien nos dio el reconocimiento legal institucional del Celaju, como un organismo no gubernamental internacional reconocido por la Cancillería de Uruguay. Enrique Iglesias había dejado la Cepal y pasó a ser canciller, luego pasó a dirigir el Banco Interamericano de Desarrollo y después pasó a ser Secretario General Iberoamericano, para coordinar la cooperación española con América Latina. Otro amigo con el que trabajábamos mucho era Juan Somavía, que pasó después a ser Director General de la OIT y, como él, muchos otros amigos ubicados en puestos claves nos apoyaban mucho. Sobre esta base, nos dimos a la tarea de crear Institutos de la Juventud, a armar Consejos de Juventud y a fortalecer las redes de investigadores especializados en los diferentes países de América Latina.

\section{F.: ¿Y funcionó?}

E. R.: La constitución de la OIJ fue la frutilla de la torta, porque le dio el marco institucional que se necesitaba para dialogar con los gobiernos. La creación de Injuves fue más difícil de lo que nos imaginamos, pero fue avanzando, sobre todo durante los años noventa. En el caso de los movimientos juveniles, Consulta Colectiva se transformó en el Foro Latinoamericano de la Juventud que tuvo marchas y contramarchas, pero ya vinculadas con la propia dinámica de los movimientos juveniles. Con la Red de Investigadores, hicimos varias reuniones, pero se nos cayeron muchos de los apoyos que teníamos, sobre todo en México y en Argentina, pero de todos modos siguió operando como Grupo de Trabajo de Clacso, sobre todo en el primer quinquenio de los años noventa. En esa época, me tocó coordinar un Equipo Técnico para sistematizar todo lo que había sobre juventud en nuestro país (entre 1992 y 1994) que culminó con la formulación de una propuesta de políticas públicas de juventud para el futuro gobierno y, por ello, cuando asumió el nuevo gobierno, me convocaron para asumir como Director Nacional del Inju para implementarla. Al mismo tiempo, asumí como presidente de la OIJ y, desde allí, conseguimos importantes apoyos para la realización de una gran Conferencia Iberoamericana sobre Salud Adolescente (la más importante de aquella época) y para replicar a escala latinoamericana la sistematización de las políticas de juventud que habíamos realizado en Uruguay. En el primer caso, el apoyo central fue el aportado por el UNFPA (cuya directora para América Latina, Marisela Padrón, era la Ministra de la Familia, donde yo había trabajado durante 1991) y, en el segundo, el apoyo clave fue el aporte del Centro Internacional de Investigaciones para el Desarrollo de Canadá. Para ambas cosas, activamos nuestras redes otra vez: la de gobiernos en el primer caso, y la de investigadores en el segundo. Fueron años muy productivos. 


\section{F.: ¿Estabas en Madrid o en Montevideo?}

E. R.: En Montevideo. La cooperación canadiense nos permitió hacer un balance sistemático de las políticas de juventud en América Latina, el primero en estas materias: veinte informes nacionales, cuatro estudios subregionales, tres evaluaciones de programas destacados y el informe final. Todo esto a mediados de los noventa, mucho antes de los libros de la OIJ y de la Cepal que vinieron después. Mi paso por el Instituto de la Juventud en Uruguay, de todos modos, fue muy corto. Me tocó asumir el cargo de gobierno, un cargo de confianza política acompañando al vicepresidente electo, pero nos fue muy mal; ganamos las elecciones, pero perdimos las internas y eso determinó que yo contaba con muy poco respaldo político para mi gestión, por lo cual, a mediados del año siguiente presenté mi renuncia, dado que no podía implementar la propuesta técnica tan rigurosamente construida en lo previo. La otra fecha clave es el 200o. Me contratan en Cepal para hacer una actualización mejorada del primer informe sistemático de la juventud que habíamos hecho en 1990 y de la evaluación de políticas de juventud que realizamos en 1995. Me trasladé a Santiago de Chile, tuve un año de fecundos y muy relevantes aprendizajes en la sede de la Cepal y el resultado fue Juventud, población y desarrollo en América Latina, el primero de una serie que comenzamos a publicar regularmente, cada tres o cuatro años. En definitiva, mi latinoamericanización tuvo ese recorrido, básicamente entre 1985 y 2014; una experiencia riquísima que me cambió la vida por completo.

\section{Treinta años después}

C. F.: Si haces un balance, serían 40 años de investigar sobre juventud: ¿cómo has visto los cambios en Uruguay, en América Latina y en el resto del mundo?, ¿qué cambio destacarías a partir de tu investigación?

E. R.: Eso sí da para una conversación larga; yo he hecho muchas comparaciones de movimientos juveniles a lo largo del tiempo. Esto que sacamos en el último libro del año pasado de las movidas juveniles, cuando en el 2005, con el apoyo del Banco Mundial, hicimos un balance juvenil de los movimientos en América del Sur (no llegamos a hacerlo en Centro América). Lo hicimos por países y por grupos de estudio; al final lo hicimos en diez ciudades. Pero en ese momento todavía teníamos una imagen bien tradicional de los movimientos juveniles; seguían siendo los de siempre: juventud estudiantil, juventud política, algunos de los sindicatos, campesinos. En paralelo había mucho sobre culturas juveniles, las tribus, digamos, yo me dediqué mucho más a los movimientos más formalizados, aunque me demoré, siempre que pasaba por ahí, por las tribus, importantísimo desde todo punto de vista. Mi impresión es que del 2005 al 2012 ya había unas diferencias enormes. 
Creo que los movimientos ahora son mucho más informales, más horizontales, más espontáneos, motivados por cuestiones de corto plazo. Es importante destacar que son diferentes a lo tradicional. En todo caso, los movimientos tradicionales, algunos vuelven y la mayor parte no lo hace y, cuando lo hacen, llegan cambiados. El movimiento chileno no es el mismo de antes; la Mane en Colombia no tiene mucho que ver con los movimientos estudiantiles de la época anterior, y me parece que ahí sí hay cambios muy notorios que hay que seguir más y mejor. Lo cierto es que estas irrupciones nuevas muestran que hay un interés enorme por lo político, por lo público, por las políticas públicas de juventud. Desde los ochenta y los noventa nosotros nos dedicamos a impulsar lo que hoy se conoce como políticas públicas integradas de juventud y descuidamos mucho las políticas sectoriales de juventud, y nos imaginamos que los institutos de juventud en los gobiernos iban a ser la octava maravilla del universo y que los consejos de la juventud también iban a serlo. 30 años después, y vista la evidencia que hay en la materia, obviamente no logramos nada de todo eso y la terca realidad nos demuestra que las políticas existentes, la más relevantes, son las políticas sectoriales de juventud, las que se hacen en salud, educación, y los Ministerios grandes como seguridad, y yo creo que ahora estamos en una nueva etapa que estamos revalorizando mucho más esto, tratando de volver desde los institutos especializados a un trabajo mucho más concentrado en modernizar y en mejorar la gestión de las grandes secretarías de Estado en juventud. Creo que es un cambio importante que está cruzado por los hechos de cabo a rabo, pero nos ha quedado la capacidad y la humildad de reconocer el fracaso (me incluyo) y creo que por ahí hay que mirar este tipo de cosas desde este otro ángulo.

La otra cosa que hay que evaluar es el campo de las investigaciones. A pesar que hemos avanzado algo, la academia sigue en deuda en el campo de las políticas de juventud, en el campo de la evaluación sistemática, rigurosa, permanente y regular de las políticas de juventud. La investigación sigue centrándose en los jóvenes y no en las políticas de la juventud y eso es una deuda enorme que tenemos todavía y tenemos que saldarla porque efectivamente hay mucho trabajo para realizar allí.

Aquí creo que había una discusión muy fuerte en el marco del neoliberalismo (sobre todo en los noventa) que todavía se ha arrastrado en este siglo y es que los investigadores que se pasaron al campo de investigación de las políticas públicas fueron contratados por organismos como el Banco Interamericano de Desarrollo, el Banco Mundial, la Unión Europea, y fueron criticados porque se pasaron al campo neoliberal —digamos_y la academia se quedó confortablemente instalada en su trinchera de pureza ideológica, dedicados a estudiar las mismas cosas de toda la vida. Esa interpretación es una simplificación absoluta. Muchos de los que trabaja- 
mos para el BID, no lo hicimos por razones económicas ni porque nos importaba en términos de estatus; no porque no somos neoliberales, pero creo que en la academia también había una tendencia a valorizar más y mejor el campo de las políticas públicas en su conjunto y eso debió reflejarse más en el campo de políticas públicas en juventud. Porque hay políticas centrales en nuestros países que deben estar evaluadas desde el punto de vista de la población y no desde el punto de vista de la gestión. Las políticas educativas, por ejemplo, hay que dejar de mirarlas desde quien enseña y pasar a mirarlas desde quien aprende y creo que, como estas, hay muchas otras por el estilo; valorarlas desde quien las implementa o desde quien las sufre -o en teoría, disfruta- no es lo mismo. Por ahí —creo- hay qué repensar este tipo de dinámicas.

Del 2000 para acá están estos informes de la OIJ con la Cepal. Como cosa destacada en el campo de las investigaciones hay mucho para analizar en términos de investigación de cada país, de cada sector. Evidentemente se ha avanzado en estas cosas: tenemos hoy en día encuestas de juventud en todos los países (algo que no teníamos), tenemos una encuesta iberoamericana que tampoco teníamos. Yo creo que se perdió mucho tiempo en el tema de la Convención Iberoamericana del derecho de los jóvenes y el resultado es algo de un valor relativo; cuestiones de la OIJ, de los periodos largos de siete u ocho años dedicados a esto. Me parece una exageración absoluta para el impacto que se dio finalmente; pero hoy en día hay un mejor equilibrio en las líneas estratégicas de la OIJ. Me parece que esto va mejorando y hay como un redescubrimiento muy fuerte en temas de los jóvenes por parte de muchos programas regionales y de varios gobiernos progresistas en América Latina.

En particular, yo diría que esto es otro campo en disputa y seguimos trabajando con una coexistencia de enfoques estratégicos muy perjudicial; predominan sin duda los enfoques de riesgo y, aunque en el discurso y en los papeles nos dediquemos a planear enfoques de derecho, ahí hay temas enormemente importantes desde el punto de vista político, institucional, etc., por trabajar a futuro. Yo creo que nos hemos equivocado en temas estratégicos antes de considerar los institutos como la octava maravilla del universo y que se llevan el mundo por delante. En el fondo hay que leerlo desde el punto de vista estratégico y para nosotros yo lo planteo siempre del mismo modo: mientras las mujeres se han dedicado a trabajar para dotar de perspectiva de género a todas las políticas públicas, en el campo de lo juvenil nos hemos dedicado a crear espacios específicos para la juventud (Parlamento Joven, Tarjeta Joven, Casa de la Juventud,...). Son espacios específicos que han aislado más de lo que han integrado. Creo que eso hay que superarlo ampliamente a futuro; hay que trabajar en términos de tratar de dotar de perspectiva generacional al conjunto de las políticas públicas. Yo creo que hace diez años yo caminaba 
en el desierto; ahora creo que digo estas cosas con más fundamento y con más evidencia, y ahora siento que tengo mucho más eco y mucha más receptividad.

Hay países claves como México que empiezan a tratar de ser consecuentes con este tipo de cambios estratégicos; una prioridad del Instituto Mexicano de la Juventud es cómo logran estructurar, cómo logran transversalizar las políticas de la juventud en todo el aparato del Estado, lo que va a llevar quién sabe cuánto tiempo, pero por lo menos el Estado tiene una acción estratégica asumida que hasta ahora no se formulaba.

C. F.: ¿Si tuvieras la oportunidad de empezar lo harías de nuevo?

E. R.: Sin ninguna duda. Uno se equivocaría menos con más experiencia en la materia, pero creo que es un tema de una relevancia enorme. Yo creo que he reunido muchas más satisfacciones que frustraciones en todo esto y sigo apostando a seguir construyendo a futuro, y a mis sesenta años sigo pensando como Benedetti: que una persona no es joven o vieja por su edad, sino por la forma en que encara su vida. Si una persona tiene más sueños que recuerdos en su cabeza es joven, independientemente de la edad que tenga, y si tiene más recuerdos que sueños en su cabeza es viejo, aunque tenga veinte años; lo dijo Mario en el último de sus libros, por cierto, dedicado a los jóvenes. Lo plantea con 83 años y remata diciendo: «Me sigo considerando absolutamente joven». Yo también, por supuesto.

Se acaba la reunión en La Plata, Argentina, el 22 de octubre del 2014.

\section{Epílogo: 2020}

C. F. (Pregunta enviada por internet el zo octubre de 2020): Ernesto, al leer la transcripción de la entrevista la veo vigente y necesaria, pero como se publicará seis años después de la conversación que tuvimos, en una situación de pandemia que pone en alerta todo lo que has creado y recordado en la entrevista, me parece inevitable plantearte a distancia la pregunta sobre cómo puede afectar el coronavirus a las políticas sobre juventud, en América Latina y más allá.

E. R. (Respuesta redactada el 5 de noviembre de 2020). En estos seis años han pasado muchas cosas, sin duda, pero las de mayor impacto y actualidad tienen que ver directamente con esta pandemia global del coronavirus, que lo ha cambiado todo, sin ningún tipo de contemplaciones. En mi caso particular, y en lo que tiene que ver directamente con lo conversado en esta entrevista, creo que los impactos son muchos y muy variados. Por un lado, mis constantes visitas de trabajo de campo a los diferentes países de la región han quedado totalmente interrumpidas, pero, a la vez, se han ampliado y mejorado significativamente las comunicaciones virtuales, 
facilitando incluso tareas que —en el modo más tradicional_ implicaban más esfuerzos y más recursos de todo tipo.

Luego de la entrevista, mi trabajo se fue concentrando cada vez más en políticas sectoriales de juventud, en línea con las reformulaciones más sustantivas que compartía en el tramo final de la misma. Se multiplicaron, de este modo, las evaluaciones programáticas concretas (en particular, en programas de inserción laboral de jóvenes y en programas de prevención de las violencias relacionadas con jóvenes, en países como El Salvador o Perú, entre otros). En paralelo, también me tocó colaborar activamente en el diseño de programas de cooperación internacional centrados en juventud, en países como Guatemala y Honduras, así como en el apoyo a la gestión en otros procesos ya más avanzados, como en México y Bolivia, entre otros. De todas estas actividades han quedado sendos informes que consignamos más adelante.

Toda esta etapa estuvo signada por grandes disputas políticas e ideológicas, con especificidades muy marcadas en el caso de países con gobiernos más progresistas o más neoliberales o conservadores, los cuales, además, cambiaron de signo político en varios casos nacionales. En países como Argentina y Brasil me tocó lidiar con cambios hacia la derecha, mientras que en países como Bolivia y Ecuador me tocó participar de procesos más estables de transformaciones progresistas, así como en otros me tocó lidiar con gobiernos más arraigados en la derecha (como en Colombia y Paraguay, entre otros). Esto, más que simplemente anecdótico, fue clave, porque me obligó a tratar de identificar hasta dónde desde una u otra de estas posturas ideológicas y políticas se hacían mejor o peor las cosas, en el campo de las políticas públicas de juventud. Fue entonces que nos dimos a la tarea de crear un índice de desarrollo de políticas de juventud, que nos permitió hacer la comparación correspondiente, constatando - finalmente-que en ambos bandos había buenas y malas experiencias, con lo cual, la correlación correspondiente no era tan fácil de establecer.

Al parecer, las verdaderas grietas o brechas en este sentido se daban y se dan al interior de los países, en el marco de encendidas disputas entre relatos fuertemente polarizados, del estilo de «estás conmigo o estás contra mí», con posturas altamente radicalizadas, centradas en la identificación del otro enemigo o culpable de todos los males de la humanidad, por decirlo muy simplificadamente. Grupos ultraconservadores que ya venían operando en casi todos nuestros países tomaron nuevos bríos con la llegada de Trump a la Casa Blanca y de Jair Bolsonaro a la presidencia en Brasil (entre otros) ligados en casi todos los casos a iglesias evangélicas, con planteos antiderechos de toda clase y tipo (contra el matrimonio igualitario, contra la interrupción voluntaria del embarazo, contra la legalización de ciertas drogas, etc.) Muchas de estas disputas se ubicaban centralmente en torno a las juventudes, y ello nos planteó nuevos desafíos 
en el campo de los estudios y las investigaciones, pero también en relación al diseño, la implementación y la evaluación de políticas públicas.

Hoy por hoy, además, todo esto se ha visto amplificado y complejizado significativamente, con el Covid-19, en particular, muchas de las aristas vinculadas con diversas violencias que afectan directa e indirectamente a las generaciones jóvenes. Por ello, en este año hemos tenido que reformular no solo las modalidades operativas de nuestro trabajo (de lo presencial a lo virtual) sino fundamentalmente en lo sustantivo, ubicando más destacadamente temas que hasta hace poco no figuraban entre las prioridades de política pública, como las vinculadas con la trata de personas, la explotación sexual y comercial, los problemas de salud mental amplificados por el encierro obligado, la clausura de espacios de socialización juvenil entre pares (ahora en proceso de reconversión total hacia lo virtual) y otros tantos por el estilo, en paralelo a los cambios que todo esto está provocando en el campo educativo (con el cierre de las escuelas y demás) y en el terreno laboral con cambios vertiginosos en relación a las múltiples transformaciones en curso en el campo tecnológico (robótica, teletrabajo, etc.) y en muchas otras áreas afines.

Creo que todos estamos obligados a repensar todo nuestro trabajo a futuro, dado que estas pandemias llegaron para quedarse y van a seguir condicionando fuertemente todas las dinámicas de nuestras sociedades a todos los niveles. Habrá que responder pertinentemente a todos estos desafíos y, para ello, con nuevas metodologías de trabajo que permitan seguir tras los objetivos de siempre, que no son otros que trabajar para que nadie quede atrás, en línea con las propuestas (por ejemplo) que acaba de formular la Cepal.

\section{Referencias y bibliografía}

Allerbeck, K., \& Rosenmayr, L. (1979). Introducción a la sociología de la juventud. Kapelusz. Rodríguez, E. (1978). Situación y perspectivas de la juventud uruguaya (No. 6). Centro de Investigación de Economía Humana.

Rodríguez, E. (1985). La juventud como movimiento social: elementos para el estudio del caso uruguayo. En C. Filgueira (Comp.), Movimientos sociales en el Uruguay de hoy. Centro de Informaciones y Estudios del Uruguay.

Rodríguez, E. (1986). Políticas de juventud en el Uruguay: balance y perspectivas. Unesco.

\footnotetext{
${ }^{3}$ La presente bibliografía, además de las referencias citadas en el texto, recopila las obras de Ernesto Rodríguez, ordenadas cronológicamente, incluyendo libros, artículos y literatura gris.
} 
Rodríguez, E. (1987). Juventud, empleo y capacitación profesional en el Uruguay: balance y perspectivas. Foro Juvenil; Fundación Ebert.

Rodríguez, E. (1988). Políticas de juventud en Argentina: balance y perspectivas. Unesco.

Rodríguez, E. (1989). Políticas de juventud en Venezuela: balance y perspectivas. Unesco.

Rodríguez, E. (1990). Políticas de juventud en Ecuador: balance y perspectivas. Unesco.

Rodríguez, E. (1991). Bases para el diseño de un plan integral de capacitación y empleo de jóvenes en Venezuela para los años noventa. Unesco.

Rodríguez, E. (1991). Primer Informe sobre la Juventud en Iberoamérica 1990 [Ponencia]. Conferencia Iberoamericana de Altas Autoridades Gubernamentales de Juventud.

Rodríguez, E. (1992). Bases para la instalación de la Red de Información Infojuve sobre Juventud en América Latina. Unesco.

Rodríguez, E. (1992). Juventud y sociedad en el Uruguay: ¿una exclusión aceptada? Inju; MEC; Fondo Nacional de Preinversión; OPP; Banco Interamericano de Desarrollo.

Rodríguez, E. (1993). Juventud y desarrollo en América Latina: bases para el diseño de planes integrales de juventud en los años noventa. Centro Latinoamericano de la Juventud.

Rodríguez, E. (1993). Políticas de juventud en el Uruguay a comienzos de los noventa. Inju; MEC; Fondo Nacional de Preinversión; OPP; Banco Interamericano de Desarrollo.

Rodríguez, E. (1994). Juventud y desarrollo en el Uruguay en los albores del Siglo XXI: propuesta de Plan Integral de Juventud 1995-200o. Inju; FAS; OPP; Banco Interamericano de Desarrollo. Rodríguez, E. (1994). Propuesta de Plan Integral de Juventud: políticas de juventud y estrategias de desarrollo en la antesala del año 200o. Inju; MEC; Fondo Nacional de Preinversión; OPP.

Rodríguez, E. (1994). Propuesta de Programa Interamericano de Capacitación Laboral y Empleo para Jóvenes 1995-200o. Organización de Estados Americanos; Centro Interamericano para el Desarrollo del Conocimiento en la Formación Profesional; Organización Internacional del Trabajo.

Rodríguez, E. (1995). Juventud y medios de comunicación en América Latina: riesgos, oportunidades y desafíos. Unesco.

Rodríguez, E. (1995). Juventud, empleo y capacitación profesional en América Latina. Centro Interamericano para el Desarrollo del Conocimiento en la Formación Profesional; Organización Internacional del Trabajo.

Rodríguez, E. (1995). Políticas de juventud en América Latina en los albores del año 20oo: logros, desafíos $y$ oportunidades. Organismo Internacional de Juventud para Iberoamérica; Centro Internacional de Investigaciones para el Desarrollo; IDRC.

Rodríguez, E. (1995). Promoción de la participación de los jóvenes en los procesos de desarrollo en América Latina y el Caribe: algunas propuestas para la acción. Banco Interamericano de Desarrollo. 
Rodríguez, E. (1996). Los jóvenes y la violencia urbana en América Latina y el Caribe: dimensiones y particularidades de un fenómeno complejo y desgarrador. Unesco.

Rodríguez, E. (1997). Mercados de trabajo, capacitación laboral y reformas estructurales en el contexto de la globalización. Programa FAS; OPP; Banco Interamericano de Desarrollo.

Rodríguez, E. (1998). Programas de capacitación y reconversión laboral en el Uruguay de fines de siglo: evaluación y propuestas para su potenciación. Programa FAS; OPP; Banco Interamericano de Desarrollo.

Rodríguez, E. (2000). Juventud, población y desarrollo en América Latina: problemas, oportunidades y desafíos. Comisión Económica para América Latina y el Caribe.

Rodríguez, E. (2002). Actores estratégicos del desarrollo: políticas de juventud para el Siglo XXI. Instituto de la Juventud.

Rodríguez, E. (2003). Políticas públicas de juventud y derechos reproductivos: limitaciones, oportunidades y desafíos en América Latina y el Caribe. United Nations Population Fund.

Rodríguez, E. (2004). Políticas públicas de/para/com as juventudes. Unesco. [En colaboración].

Rodríguez, E. (2004). Políticas públicas y marcos legales para la prevención de la violencia relacionada con jóvenes: estado del arte en América Latina 1995-2004. GTZ; Organización Panamericana de la Salud.

Rodríguez, E. (2005). Juventud, desarrollo y democracia en América Latina. Revista Nueva Sociedad (200), 52-70.

Rodríguez, E. (2005). Organizaciones y movimientos juveniles en América del Sur: estado de situación y bases para un programa de fortalecimiento institucional. Banco Mundial; Unesco.

Rodríguez, E. (2006). Apoyo al fortalecimiento del Instituto Nacional de la Juventud de Honduras y de sus prioridades estratégicas. Naciones Unidas.

Rodríguez, E. (2006). Estrategias para la inserción laboral de jóvenes en desventaja: la experiencia del Programa Entra 21 en América Latina y el Caribe. IYF; Fondo Multilateral de Inversiones; Banco Interamericano de Desarrollo.

Rodríguez, E. (2006). Jóvenes en cifras: Paraguay 2005. United Nations Population Fund.

Rodríguez, E. (2006). Jóvenes y violencias en América Latina: priorizar la prevención con enfoques integrados. Centro Latinoamericano de la Juventud.

Rodríguez, E. (2006). Jóvenes y violencias en El Salvador: situación, perspectivas y políticas públicas. Unesco.

Rodríguez, E. (2006). Jóvenes y violencias en Nicaragua: avances recientes y desafíos a encarar desde la dinámica de las políticas públicas. Unesco. 
Rodríguez, E. (2006). Prevención y atención de las violencias relacionadas con jóvenes en América Latina: una herramienta clave para la convivencia y la seguridad ciudadana a nivel local. Programa de las Naciones Unidas para el Desarrollo.

Rodríguez, E. (2006). Políticas públicas de juventud en Honduras: desafíos y propuestas en el comienzo de una nueva gestión de gobierno. Naciones Unidas.

Rodríguez, E. (2007). Enfoques y herramientas pertinentes: condiciones básicas para el éxito de las políticas públicas de juventud en América Latina. Centro Latinoamericano de la Juventud.

Rodríguez, E. (2007). Guatemala: Misión de Evaluación Política y Estrategia. Programa de apoyo presupuestario sectorial: sector juventud. Unión Europea. [En colaboración con Giovani Caprio].

Rodríguez, E. (2007). Jóvenes, violencias y gobernabilidad democrática: algunos puntos clave para la toma de decisiones [Ponencia]. Primer Foro de Alto Nivel de Ministros a Cargo de las Áreas de Juventud de Centroamérica. Unesco.

Rodríguez, E. (2008). Desarrollo juvenil y prevención de la violencia en Ecuador: documento de proyecto (incluyendo su marco lógico). Unesco.

Rodríguez, E. (2008). Políticas de juventud y cohesión social en América Latina: logros obtenidos y desafíos a encarar. Comisión Económica para América Latina y el Caribe; Secretaría General Iberoamericana; Organismo Internacional de Juventud para Iberoamérica.

Rodríguez, E. (2008). Políticas públicas de juventud en El Salvador: logros y limitaciones del período 2004-2008. Secretaría Nacional de la Juventud; GTZ.

Rodríguez, E. (2008). Políticas públicas de juventud en América Latina: experiencias adquiridas y desafíos a encarar. Pensamiento Iberoamericano, (3), 273-291.

Rodríguez, E. (2008). Programa de apoyo a las políticas de juventud en Guatemala 2009-2012, bajo la modalidad de apoyo presupuestario sectorial. Unión Europea.

Rodríguez, E. (2008). Programa de apoyo a los niños y jóvenes de la calle en Colombia: evaluación final. Unión Europea.

Rodríguez, E. (2008). Propuesta de Plan Nacional de Prevención de la Violencia para el Período 20102030 y Plan de Acción 2008-2009. PNPRRS.

Rodríguez, E. (2008). Salud sexual y reproductiva y políticas públicas de juventud: experiencias y desafíos en América Latina. Comisión Económica para América Latina y el Caribe.

Rodríguez, E. (2009). Generación y gestión del conocimiento sobre políticas de juventud en América Latina: avances concretados y desafíos a encarar. Universidad Javeriana.

Rodríguez, E. (2009). Politiques Publiques de Jeunesse en Haïti: Chemins Parcourus et Defís a Relever. United Nations Population Fund; MJSAC.

Rodríguez, E. (2010). Jóvenes y ética en el ciberespacio: un análisis del estado del conocimiento en América Latina y el Caribe (2000-2010). Unesco. 
Rodríguez, E. (2010). Política Pública de Juventud 2010-2015: construyendo una nación pluricultural, incluyente y equitativa; construyendo la unidad en la diversidad y el desarrollo con identidad. Programa de Fortalecimiento del Sector Juventud.

Rodríguez, E. (2010). Políticas Públicas de Juventud en América Latina: avances concretados y desafíos a encarar en el marco del Año Internacional de la Juventud. Unesco.

Rodríguez, E. (2010). Políticas Públicas de Juventud en América Latina: de la crisis del modelo renovado a la renovación impostergable del modelo. Seminario Internacional Jóvenes Construyendo Mundos. Universidad Complutense; Instituto de la Juventud.

Rodríguez, E. (2010). Políticas públicas de Juventud: aprendizajes y desafíos en América Latina [Ponencia]. Foro Parlamentario Mundial sobre Juventud (Ciudad de México) y Conferencia Mundial de la Juventud (Guanajuato, México).

Rodríguez, E. (2011). Evaluación de medio término del Programa Entra 21, Fase 2: informe final. ARS; IYF; Fondo Multilateral de Inversiones; Banco Interamericano de Desarrollo. [En colaboración con Juan Buchet y Martha Romero].

Rodríguez, E. (2011). Juventud y cohesión social en el Uruguay: ¿de la invisibilidad al protagonismo? Centro de Investigación de Economía Humana; Secretaría General Iberoamericana.

Rodríguez, E. (2011). Políticas de juventud en Centroamérica: construyendo un paradigma para el desarrollo social. Organismo Internacional de Juventud para Iberoamérica.

Rodríguez, E. (2011). Políticas públicas de juventud y desarrollo social en América Latina: bases para la construcción de respuestas integradas. Unesco.

Rodríguez, E. (2012). Jóvenes ecuatorianos en cifras: indicadores claves de la década 2001-2010 y proyecciones al 2050. Programa de las Naciones Unidas para el Desarrollo; Organización Internacional para las Migraciones; Organización Internacional del Trabajo; Organismo Internacional de Juventud para Iberoamérica; United Nations Population Fund; Unicef.

Rodríguez, E. (2012). Prevención de la violencia con participación juvenil (Projóvenes II). Informe final de la evaluación de medio término. ARS; Consejo Nacional de la Juventud; Unión Europea. [Con José María Ríos].

Rodríguez, E. (2012). Salud pública, políticas sociales y desarrollo humano de adolescentes y jóvenes indígenas en América Latina: experiencias acumuladas y desafíos a encarar. Organización Panamericana de la Salud.

Rodríguez, E. (2012). Trabajo decente, seguridad ciudadana y desarrollo: bases conceptuales para un estado del conocimiento con énfasis en jóvenes de Centroamérica y Panamá. Organización Internacional del Trabajo.

Rodríguez, E. (2013). Agenda de desarrollo e inversión social en juventud: una estrategia post 2015 para Iberoamérica. Organismo Internacional de Juventud para Iberoamérica. 
Rodríguez, E. (2013). Diseño, implementación, monitoreo y evaluación de planes departamentales de juventud: una guía para la acción. Unión Europea.

Rodríguez, E. (2013). Jóvenes, violencias y cultura de paz en América Central: enfoques, dilemas y respuestas a desplegar en el futuro. Unesco.

Rodríguez, E. (2013). Jóvenes, violencias y cultura de paz en América Central: enfoques, dilemas y respuestas a desplegar en el futuro. Unesco.

Rodríguez, E. (2013). Movimientos juveniles en América Latina: entre la tradición y la innovación. Secretaría Nacional de la Juventud; Centro Latinoamericano de la Juventud; Unesco. [En colaboración].

Rodríguez, E. (2013). Niños, niñas y adolescentes en América Latina: situación actual, políticas públicas vigentes y principales desafíos a encarar en el mediano plazo. Térre des Hommes.

Rodríguez, E. (2013). Políticas de inclusión social de jóvenes en América Latina: situación, desafíos y recomendaciones para la acción. Clacso; Unesco. [En colaboración]

Rodríguez, E. (2014). Jóvenes ecuatorianos en España: dilemas y certezas de una generación. Organismo Internacional de Juventud para Iberoamérica. [En colaboración]

Rodríguez, E. (2014). Mujeres jóvenes, empleo y sociedad red en América Latina: vulnerabilidades, oportunidades y desafíos a encarar. Centro Latinoamericano de la Juventud.

Rodríguez, E. (2014). Políticas de juventud en América Latina: de la irrelevancia a la incidencia. Enciclopedia de la Juventud; SIj; Universidad Nacional Autónoma de México.

Rodríguez, E. (2015). Bases para la construcción de un índice de desarrollo de políticas sectoriales de juventud en América Latina. Centro Latinoamericano de la Juventud; Unesco; Organismo Internacional de Juventud para Iberoamérica.

Rodríguez, E. (2015). Políticas públicas de juventud en Honduras: bases para una Estrategia de Implementación 2015-2018. Organismo Internacional de Juventud para Iberoamérica.

Rodríguez, E. (2015). Políticas públicas de juventud en Paraguay: bases para el diseño de un Plan de Acciones Integradas para el Período 2015-2018. Banco Interamericano de Desarrollo; United Nations Population Fund.

Rodríguez, E. (2015). Subjetividades diversas: análisis de la situación política, social y económica de las juventudes peruanas. Centro Latinoamericano de la Juventud; Secretaría Nacional de la Juventud; Unesco. [En colaboración].

Rodríguez, E. (2016). Inversión social, diseños institucionales y logros obtenidos en políticas públicas de juventud en América Latina y el Caribe: la excepcionalidad cubana en el contexto regional. Centro Latinoamericano de la Juventud; Organismo Internacional de Juventud para Iberoamérica. 
Rodríguez, E. (2016). Políticas públicas de juventud en el Perú 200o-2021: camino recorrido y desafíos a encarar. Centro Latinoamericano de la Juventud; Organismo Internacional de Juventud para Iberoamérica.

Rodríguez, E. (2016). Políticas públicas de juventud en el salvador: un caso ejemplar a tener centralmente en cuenta. Unión Europea.

Rodríguez, E. (2016). Políticas públicas de juventud en Panamá: haciendo camino al andar. Centro Latinoamericano de la Juventud; Organismo Internacional de Juventud para Iberoamérica. Rodríguez, E. (2016). Políticas públicas de juventud, administración pública y reforma del Estado en Iberoamérica: experiencias acumuladas y desafíos a encarar. Secretaría General Iberoamericana; Centro Latinoamericano de Administración para el Desarrollo.

Rodríguez, E. (2017). ¿Radicalización juvenil potenciada o facilitada por internet? Comparando procesos en Europa y América Latina, a propósito del vínculo entre jóvenes y terrorismo islamista. Clacso, Unesco.

Rodríguez, E. (2018). Políticas de/para/con juventudes de Honduras 2018-203o. Instituto Nacional de la Juventud; Programa de las Naciones Unidas para el Desarrollo.

Rodríguez, E. (2018). Propuesta de Diplomado en Gestión Pública y Políticas de Juventudes. United Nations Population Fund; EGPP, Escuela de Gestión Pública Plurinacional de Bolivia.

Rodríguez, E. (2019). Desigualdades, miedos, intolerancias, odios y violencias en América Latina: respondiendo al desafío de la radicalización juvenil en plena era de internet. Unesco; Organismo Internacional de Juventud para Iberoamérica.

Rodríguez, E. (2019). Juventudes, políticas públicas y mundo del trabajo en América Latina: dilemas y desafíos en tiempos de intensas disputas ideológicas y políticas. Centro Latinoamericano de la Juventud.

Rodríguez, E. (2019). Pensar las políticas públicas de juventud desde las narrativas juveniles, en el marco de las miradas neoliberales, neoconservadoras y neodesarrollistas sobre las y los jóvenes, vigentes y en disputa en América Latina. Centro Latinoamericano de la Juventud.

Rodríguez, E. (2020). Adolescentes y jóvenes «invisibilizados» en el Perú: situación actual y desafíos a encarar en tiempos de pandemia. Oficina de las Naciones Unidas contra la Droga y el Delito.

Rodríguez, E. (2020). Juventudes rurales y políticas públicas en América Latina con foco en Argentina: Estado del Conocimiento 2000-2020. Iica.

Rodríguez, E. (2020). Perspectiva generacional y políticas de empleo en América Latina y el Caribe: propuesta de nota conceptual de la OIT. Organización Internacional del Trabajo.

Solari, A. (1971). Algunas reflexiones sobre la juventud latinoamericana. Instituto Latinoamericano de Planificación Económica y Social. 